\title{
OTIMIZAÇÃO EM TEMPO DE EXECUÇÃO DE CONTROLADORES USANDO HARDWARE COMPUTACIONAL NA CONFIGURAÇÃO MESTRE-ESCRAVO
}

\author{
Alexandre Rodrigues Mesquita \\ Divisão de Engenharia Eletrônica \\ Instituto Tecnológico de Aeronáutica \\ 12.228-900 São José dos Campos - SP
}

\author{
Karl Heinz Kienitz \\ Divisão de Engenharia Eletrônica \\ Instituto Tecnológico de Aeronáutica \\ 12.228-900 São José dos Campos - SP
}

\begin{abstract}
This work describes the potential and the flexibility of online controller optimization strategies, using computational hardware in master-slave configuration, with use of the xPC Target tool. This is an inexpensive alternative to the use of DSP solutions. The control algorithm runs on the slave PC, while the real time optimization runs on the master PC, which modifies parameters in the slave to optimize the dynamic response of the controlled plant. To illustrate methods and procedures parametric optimization strategies without derivative use were adapted and compared in the scope of a magnetic levitation control system. Special attention was given to techniques to speed up performance function calculation and to prevent (temporary) instability of the controlled system.
\end{abstract}

KEYWORDS: control; host-target environment; magnetic levitation; numerical methods; parametric optimization; xPC Target.

\section{RESUMO}

Este trabalho descreve o potencial e a flexibilidade de estratégias de otimização de controladores feita em tempo de execução, usando um hardware computacional na configuração mestre-escravo, com emprego da ferramenta

Artigo Submetido em 06/11/03

1a. Revisão em ; 16/02/04

2a. Revisão em;01/06/04

Aceito sob recomendação do Ed. Assoc. José R. C. Piqueira comercial xPC Target, que representa uma alternativa de baixo custo ao uso de placas de DSP. O controle é realizado no computador escravo, enquanto a otimização é executada no computador mestre, que altera parâmetros no escravo buscando otimizar a resposta dinâmica da planta controlada. Para ilustrar procedimentos e metodologias, foram adaptadas e comparadas estratégias de otimização paramétrica, baseadas em algoritmos numéricos que não usam derivada, para controladores de um sistema de levitação magnética. Foi dada especial atenção à pesquisa de técnicas para acelerar o cálculo da função de desempenho e para evitar que o procedimento de otimização comprometesse, ainda que temporariamente, a estabilidade do sistema controlado.

PALAVRAS-CHAVE: algoritmos numéricos; controle; hardware mestre-escravo; levitação magnética; otimização paramétrica; xPC Target.

\section{INTRODUÇÃO}

O controle digital de sistemas dinâmicos através de controladores dedicados é hoje algo comum. Tradicionalmente um núcleo de sistema operacional de tempo real e placas com DSPs e conversores A/D e D/A têm sido usadas em computadores tipo PC para executar programas que implementam os controladores dedicados. A desvantagem dessa solução é seu custo. Uma alternativa é o uso de dois PCs na configuração mestre-escravo. O PC escravo (sem periféricos) é mais barato do que uma placa de DSP de alto desempenho, fornece recursos computacionais comparáveis e permite a execução dos mesmos algoritmos. O PC escravo executa tarefas de controle e o PC mestre pode ser usado para outras tarefas. 
Em especial é possível utilizá-lo para otimizar os procedimentos de controle adotados no PC escravo, informando a este em tempo de execução as alterações paramétricas indicadas para uma melhoria do desempenho. A desvantagem da configuração mestre-escravo é o hardware menos compacto.

O objetivo deste trabalho é ilustrar o potencial e a flexibilidade de estratégias de otimização implementadas no PC mestre que utilizam dados colhidos pelo PC escravo. Optou-se por uma apresentação usando estratégias de otimização de controladores para um sistema de levitação magnética (Feedback Instruments Ltd., 1997), hardware mestre-escravo e as ferramentas integradas xPC Target Real Time Workshop (RTW) - MATLAB / Simulink e um compilador $\mathrm{C} / \mathrm{C}++$. A otimização discutida é do tipo paramétrica, baseada em algoritmos numéricos que não usam gradientes, tendo sido testadas e investigadas várias estratégias. A planta dinâmica utilizada é instável em malha aberta e muito sujeita a variação em função de perturbações e condições de operação.

Este trabalho tem a seguinte estrutura. Nas seções 2 e 3 são apresentados a estrutura de hardware computacional mestre-escravo e o sistema de levitação magnética, respectivamente. Na seção 4 discute-se a otimização de controladores. Conclusões e comentários são dados na seção 5 .

\section{O HARDWARE MESTRE-ESCRAVO}

A figura 1 apresenta um diagrama da configuração mestreescravo para controle de sistemas dinâmicos. Uma ferramenta apropriada para emprego nesse hardware é o xPC Target, que permite a geração de códigos otimizados, portáteis e padronizados a partir dos modelos do Simulink, valendo-se do RTW, que por sua vez utiliza um compilador $\mathrm{C} / \mathrm{C}++$ em retaguarda, invocando-o de forma invisível ao usuário. Esses códigos são carregados ao PC escravo, que executa um núcleo de sistema operacional de tempo real de 32 bits extremamente compacto. Esse núcleo de sistema operacional é carregado pelo escravo durante sua inicialização, por exemplo a partir de um disco flexível gerado no PC mestre. A comunicação entre os dois PCs pode realizar-se via interface serial ou interface TCP/IP. Neste trabalho foi utilizada a interface serial RS-232. Visto que o PC escravo é dedicado a executar o código gerado, não possuindo periféricos, mas dispondo de muita memória, o xPC Target viabiliza desempenho e estabilidade aumentados a custo reduzido. Para estabelecer comunicação com o mundo físico o PC escravo dispõe de conversores $\mathrm{A} / \mathrm{D}$ e D/A, que são interpretados como blocos de entrada e saída no ambiente Simulink. Assim, ele poderá ser usado no controle de sistemas dinâmicos. O monitor do PC mestre e o monitor opcional do PC escravo podem ser utilizados para a visualização de sinais e eventos. Além do controle de sistemas dinâmicos, a configuração apresentada na figura 1 presta-se também para a simulação em tempo real de sistemas dinâmicos, notadamente simulação HIL ("hardware-in-the-loop").

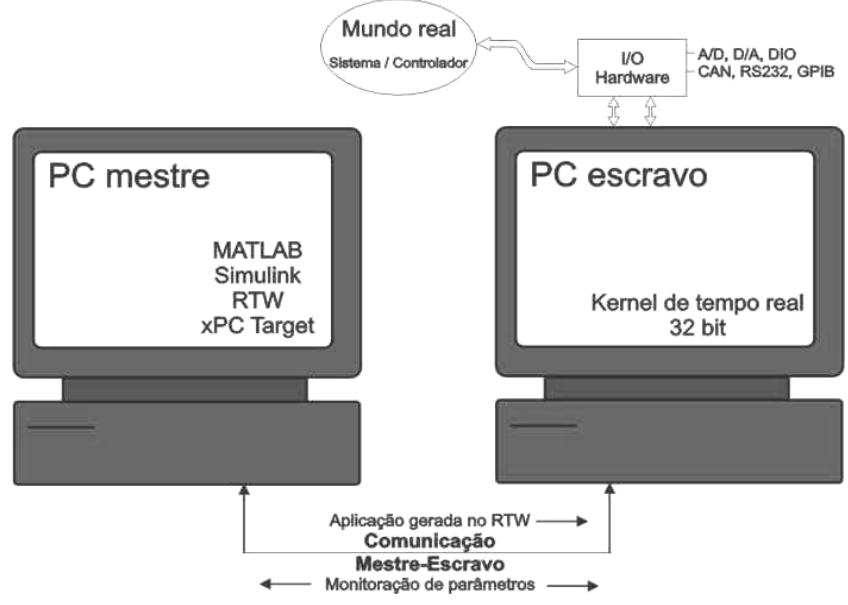

Figura 1 - Configuração de hardware mestre-escravo para controle de sistemas dinâmicos

\section{O SISTEMA DE LEVITAÇÃO MAGNÉTICA}

A planta dinâmica utilizada neste trabalho é um sistema de levitação magnética, disponível como kit de laboratório (Feedback Instruments Ltd., 1997). Embora esse equipamento seja simples e compacto, trata-se de um sistema complexo visto da perspectiva da engenharia de controle, por apresentar não-linearidades, instabilidade em malha aberta e por não ser estabilizável por controle proporcional. O levitador magnético utilizado está representado esquematicamente na figura 2.

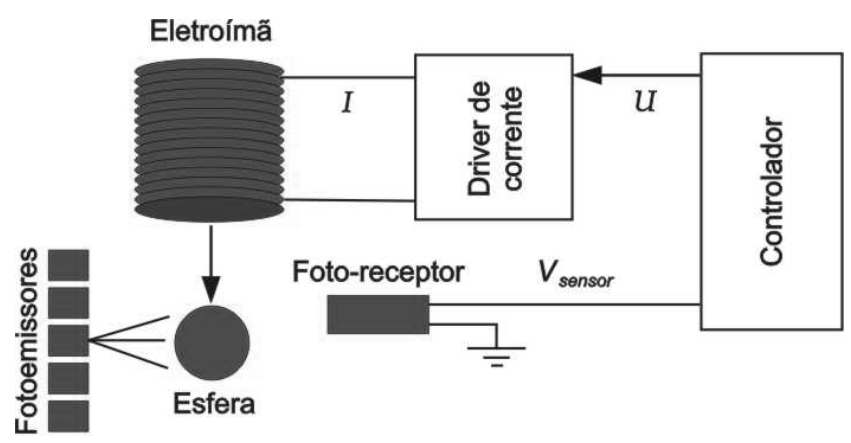

Figura 2 - Representação esquemática do levitador magnético

A levitação ocorre pela compensação da força gravitacional através da força eletromagnética exercida pelo etetroímã. Há um fotossensor a infravermelho que retorna uma tensão proporcional à altura da sombra da esfera. Essa tensão é utilizada por um controlador que impõe a tensão de controle ao driver de corrente que, por sua vez, alimenta a bobina. 
Sendo $H$ a distância da esfera ao eletroímã, $g$ a aceleração da gravidade, $m$ a massa da esfera, $I$ a corrente no eletroímã, em primeira aproximação, pode-se estabelecer a partir do princípio da mínima relutância o seguinte modelo (Galvão et al., 2003), onde $k$ é um parâmetro. Observações experimentais detalhadas levam à conclusão de que $k$ depende fracamente de $H$ (Grimm, 2002).

$$
\frac{d^{2} H}{d t^{2}}=g-\frac{k}{m} \frac{I^{2}}{H^{2}}
$$

O uso de um modelo derivado por linearização da equação acima (supondo $k$ constante) foi proposto pelo próprio fornecedor do kit, Feedback Instruments Ltd. (1997). O modelo linearizado pode ser expresso como função de transferência entre a variação da tensão de controle e a variação da tensão na saída do fotossensor, que depende linearmente de $H$ :

$$
G(s)=\frac{\alpha \gamma}{m s^{2}-\lambda}
$$

Os parâmetros $\alpha \gamma$ e $\lambda$ desta função de transferência são de difícil determinação, pois não dependem apenas do ponto de operação (definidos pelos valores nominais de $I$ e $H$ ), mas variam (lentamente, via $k$ ) com o tempo, em função de situações "exógenas" tais como as condições de magnetização da esfera (Galvão et al., 2003). O modelo linear não permite explicar ciclos-limite de baixa amplitude que ocorrem em torno da maioria dos pontos de operação para muitos controladores lineares. Assim sendo, é difícil, a partir do modelo linear, projetar-se um controlador que garanta desempenho robusto ao sistema.

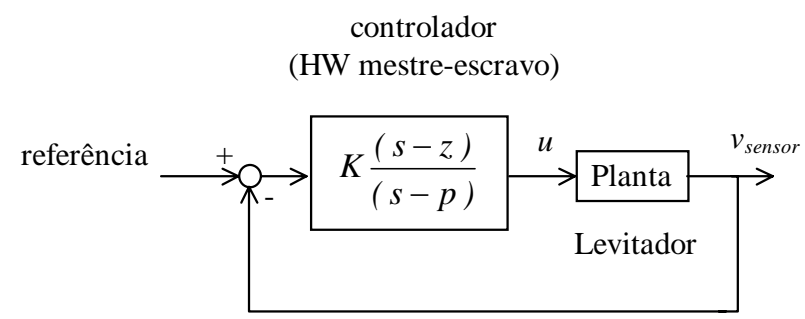

Figura 3 - Diagrama de blocos para o sistema de levitação magnética controlado

A figura 3 mostra o diagrama de blocos da planta com um controlador linear de primeira ordem que foi implementado via Simulink / xPC Target com método de integração Runge-Kutta de $4^{\underline{a}}$ ordem e passo de integração de 0,5 [ms] no hardware mestre-escravo. Os valores iniciais de $z$ e $p$ forma escolhidos de forma a ter-se um compensador de avanço de fase. Durante a otimização os parâmetros $K, z$ e $p$ foram ajustados em busca de uma lei de controle de desempenho ótimo segundo critérios discutidos na seção seguinte.
Uma fotografia do conjunto de laboratório em funcionamento encontra-se na figura 4.

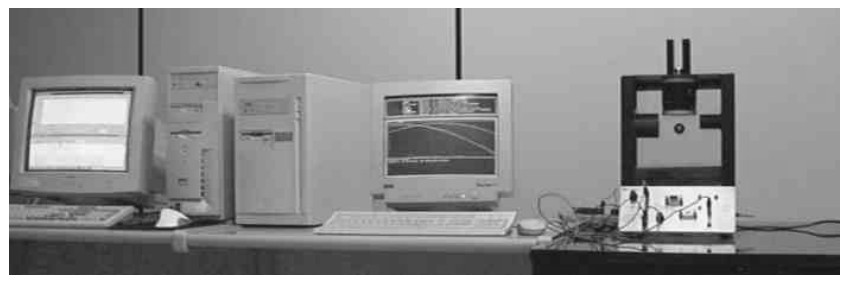

Figura 4 - Hardware em configuração mestre-escravo controlando um sistema de levitação magnética

A figura 5 ilustra o funcionamento do sistema, apresentando a leitura de um dos instrumentos virtuais usados na monitoração de variáveis relevantes. Neste gráfico observa-se a forma de onda da posição da esfera medida em [V] (volts) pelo sensor. As formas anteriores a t = 5 [s] mostram a colocação da esfera no levitador; a tensão de saturação de 2 [V] indica que a esfera não se encontra dentro do alcance do sensor. Os tempos de execução de tarefa do controlador (tempo que o PC escravo leva para efetuar aquisição de dados e os cálculos) tiveram valor máximo de 0,14 [ms] e valor médio de 0,12 [ms].

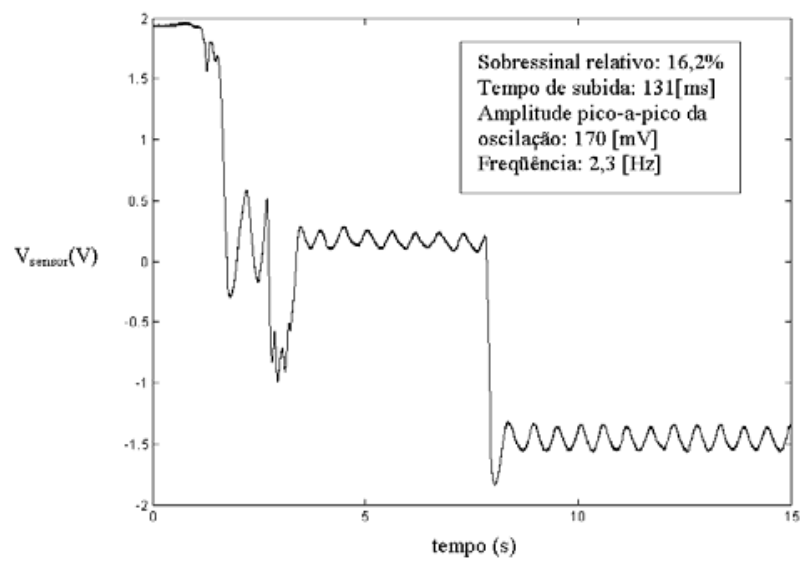

Figura 5 - Forma de onda da resposta a degrau (aplicado em tempo $=9[\mathrm{~s}]$ ) do sistema controlado

Um dos problemas práticos para o controle é a variação temporal da magnetização do sistema, que se traduz numa variação paramétrica não quantificada neste trabalho. Outro problema de relevância é o ciclo-limite observado na figura 5, que se deve a não-linearidades da dinâmica, negligenciadas no modelo linear. Confirmou-se que sintonia apropriada dos parâmetros do controlador reduz a amplitude dessa oscilação.

\section{OTIMIZAÇÃO DO CONTROLADOR}

Para realizar a otimização do controlador em tempo de execução dispôs-se de um programa principal em formato de arquivo "M-file". Este inicia o controle do levitador 
magnético através da execução em tempo real pelo escravo de um programa gerado pelo xPC Target a partir de um modelo Simulink, que implementa a lei de controle escolhida. Foi necessário que o controlador utilizado nos experimentos fosse descrito em um diagrama de blocos Simulink tal que parâmetros de otimização correspondessem a ganhos, que são grandezas reajustáveis em tempo de execução no escopo do xPC Target, ao contrário dos parâmetros de outros blocos do Simulink (como zero-pole ou transfer function).

Conforme indicado na figura 3 , a lei de controle utilizada neste estudo foi:

$$
C(s)=K \frac{s-z}{s-p}
$$

sendo os parâmetros $K$, z e $p$ escolhidos para otimização.

No programa principal é acionado um procedimento de minimização baseado em algoritmo sem uso de derivadas. Esse algoritmo, por sua vez, repetidas vezes invoca a rotina que calcula a função de desempenho, gerando e testando valores para os parâmetros de otimização. O procedimento de minimização modifica em tempo de execução os valores dos parâmetros no controlador ( $K, z$ e $p$ ) e calcula a função de desempenho desejada com base num conjunto de amostras da resposta a degrau do sistema controlado. Para se obter tal resposta a planta é excitada por uma onda quadrada de pequena amplitude injetada em programa. Os dados adquiridos através dos conversores são transferidos do PC escravo ao PC mestre.

Os procedimentos numéricos de otimização foram implementados em linguagem MATLAB. Foram implementados / utilizados os seguintes procedimentos:

- $\quad$ método de Rosenbrock;

- método de Rosenbrock com recozimento simulado;

- $\quad$ método de Powell;

- método dos poliedros flexíveis;

- algoritmo cúbico (caso de constante Lipschitz desconhecida);

- procedimento fminsearch do Optimization Toolbox do MATLAB.

Três dos métodos implementados são tradicionalmente tratados pela literatura especializada (Himmelblau, 1972; Murray et al., 1981): o de Rosenbrock (1960), o de Powell (1964) e o dos poliedros flexíveis (Spendley et. al., 1962). Para a implementação seguiu-se a descrição apresentada por Himmelblau (1972). Os algoritmos de Rosenbrock e Powell foram implementados com a busca unidimensional de Davis-Swann-Campey (Swann, 1964), implementada conforme Nascimento e Yoneyama (2000). A adaptação da técnica de recozimento simulado para o método de Rosenbrock seguiu Nascimento e Yoneyama (2000), e teve por objetivo robustecer o método de Rosenbrock contra a atração por mínimos locais. O algoritmo cúbico, descrito por Galperin (1993), foi o único método implementado com garantia de convergência para mínimos globais. Uma alternativa adicional seria o uso de algoritmos genéticos, cuja implemantação não foi feita no contexto do trabalho aqui descrito.

\subsection{A Função de Desempenho}

Tomando sobressinal relativo percentual $\left(M_{p}\right)$, tempo de subida ( $t_{s}$, medido em [ms]) e amplitude pico-a-pico da oscilação do ciclo limite ( $A$, medida em $[\mathrm{mV}])$ como indicadores de desempenho, escolheram-se funções semelhantes à norma de um vetor para medir o desempenho do controle realizado. Desse modo a minimização da função implica minimizar cada um desses parâmetros. A contribuição de cada componente foi elaborada normalizando-se o valor do indicador de desempenho usando um valor desejado estipulado com base em experimentos anteriores e atribuindo-lhe determinado peso conforme sua importância relativa na percepção do projetista do que seria uma "boa resposta" do sistema. Assim, arbitraram-se as seguintes funções de desempenho dadas em (2)-(4).

$$
\begin{gathered}
f_{1}=50\left(\frac{M_{p}}{12}\right)^{2}+30\left(\frac{A}{139}\right)^{2}+20\left(\frac{t_{s}}{111}\right)^{2} \\
f_{2}=50\left|\frac{M_{p}}{12}\right|+30\left|\frac{A}{139}\right|+20\left|\frac{t_{s}}{111}\right| \\
f_{3}=100 \max \left[\left|\frac{M_{p}}{12}\right|,\left|\frac{A}{139}\right|,\left|\frac{t_{s}}{111}\right|\right]
\end{gathered}
$$

Os valores que dividem $M_{p}, t_{s}$, e $A$, são constantes de escalonamento escolhidas em função dos valores típicos que essas variáveis podem assumir.

Outro indicador de desempenho com eventual interesse, aqui não considerado, seria o erro de regime para entrada degrau.

Deve-se ressaltar que o tempo de cálculo da função é alto, pois é analisada a resposta a meio período da onda quadrada injetada na planta, e tal período deve ser grande $(\approx 10$ s) para que a posição da esfera possa estabilizar-se entre a aplicação de um e outro degrau.

Como os métodos de minimização usados não impõem restrições à procura em determinada região do espaço de parâmetros, é possível ter-se que avaliar a função de 
desempenho em pontos $(K, z, p)$ que correspondem a um sistema de malha fechada instável. Quando isto ocorre, há saturação na tensão do sensor, permitindo que o programa identifique o fato e atribua valor infinito à função. No entanto não é prático aceitar que durante a otimização o sistema possa ser levado à instabilidade. Isso requereria a recolocação da esfera cada vez que ela caísse ou se chocasse contra o eletroímã. Concebeu-se, portanto, uma forma de aumentar a automação do processo: a análise preliminar da estabilidade usando um modelo do sistema. No caso de conclusão pela instabilidade durante essa análise, o ponto não seria testado e a função de desempenho receberia valor infinito.

Inicialmente procurou-se usar um modelo não-linear do mesmo aparelho levantado por Grimm (2002). Esse modelo, no entanto, não se mostrou útil na previsão da instabilidade do sistema, pela dificuldade em determinar as bacias de atração. Por outro lado, uma previsão (via análise) usando um modelo linearizado da planta também não se mostrou efetiva na predição de instabilidade, uma vez que para usar parâmetros representativos do modelo é preciso conhecer seu ponto de operação e estabelecer hipóteses sobre a magnetização da esfera. Tentativas de estimar o ponto de operação a partir da posição média da esfera na iteração anterior não tiveram valor prático. Sem embargo, Grimm (2002) já havia alertado para erros consideráveis do modelo advindos da variação temporal da magnetização da esfera e do eletroímã. Por esse motivo abandonou-se, por fim, a tentativa de usar análise (previsão) de estabilidade no ciclo de otimização.

Outra solução para evitar que a estratégia de otimização tente avaliar o desempenho do sistema em pontos $(K, z, p)$ inadequados do ponto de vista de estabilidade, seria alterar os algoritmos de otimização de modo que dessem passos pequenos o suficiente para não se aproximarem de pontos de instabilidade. Entretanto, esse tipo de solução causou um grande aumento no tempo de otimização e também foi abandonado. Optou-se portanto em tolerar a instabilidade, reconhecendo como mais adequadas aquelas estratégias que, naturalmente, não provocassem quedas freqüentes da esfera durante a otimização.

\subsection{Experimentos de Otimização com o Controlador do Levitador Magnético}

Todos os experimentos foram realizados fornecendo-se ao MAGLEV a referência 1,53 [V] para a posição da esfera. Na implementação do controlador usando Simulink / xPC Target, o método de integração utilizado foi o de RungeKutta de $4^{\mathrm{a}}$ ordem com passo de integração de 0,5 [ms]. A amplitude da onda quadrada para perturbar a esfera foi de 0,1 [V], com período de 10 [s]. A amostragem de pontos para calcular a função de desempenho durou 5 [s] e ocorreu durante a subida da esfera (semi-ciclo positivo do sinal); foram tomadas 100 amostras por segundo. O cálculo da função de desempenho em cada ponto avaliado durou 10 [s].

Todos os métodos de otimização foram inicialmente utilizados para minimizar uma função teste com a finalidade de verificar o funcionamento dos algoritmos implementados. Posteriormente cada um dos métodos foi usado na experimentação com o controlador do levitador magnético. Nessa experimentação foram bem sucedidos apenas os métodos de Rosenbrock, Rosenbrock com recozimento simulado e de Powell. Os demais algoritmos foram mal sucedidos, na medida em que:

- regularmente ocorreram variações excessivas dos parâmetros do controlador, com quedas da esfera ou sua colisão com o eletroimã; ou

- no caso do método dos poliedros flexíveis e da busca fminsearch do MATLAB Optimization Toolbox, não obstante as inúmeras combinações testadas para seus parâmetros que não provocavam quedas da esfera ou sua colisão com o eletroímã, as buscas convergiram sempre para o centro do poliedro inicial, levando a um resultado insatisfatório. (O comportamento ineficaz desses métodos para funções quadráticas é conhecido na teoria.)

Não se conseguindo um mecanismo que pudesse prever a queda da esfera ou sua colisão com o eletroímã para um conjunto de parâmetros do controlador, tornou-se inviável o uso do algoritmo cúbico. Durante a execução desse algoritmo as quedas ou colisões são freqüentes, pois o algoritmo não realiza uma busca descendente, mas amostra a função de desempenho num cubo do espaço de parâmetros.

De modo geral, a otimização pode ser considerada satisfatória com os métodos de Rosenbrock, Rosenbrock com recozimento simulado e de Powell, tendo na segunda e na terceira iterações atingido valores muito abaixo daqueles relativos aos pontos iniciais de busca. Não se pôde falar em mínimo global, visto que os métodos não o garantem. A partir de certa iteração, nota-se oscilação dos valores da função desempenho devido à mudança das condições de magnetização da esfera, ocasionada em parte pelo próprio processo de otimização, quando se promovem mudanças bruscas no valor da variável de controle. Tal fato é em parte responsável pelo observado nos gráficos das figuras 6 a 8, que retratam a evolução por cálculo de função e não por iteração do algoritmo de otimização. Os valores iniciais diferentes de $f_{1}$ na figura 6 devem-se à realização das otimizações em ocasiões diferentes, ilustrando uma vez mais a dependência da dinâmica das condições de magnetização existentes. Uma outra manifestação dessa dependência é a variação dos valores dos minimizadores 
dos diversos experimentos que foi de até $15 \%$ para $p$, de até $18 \%$ para $z$ e de até $65 \%$ para $K$. Os valores médios dos minimizadores foram $K=35, p=-445[\mathrm{rad} / \mathrm{s}], z=-40$ $[\mathrm{rad} / \mathrm{s}]$.

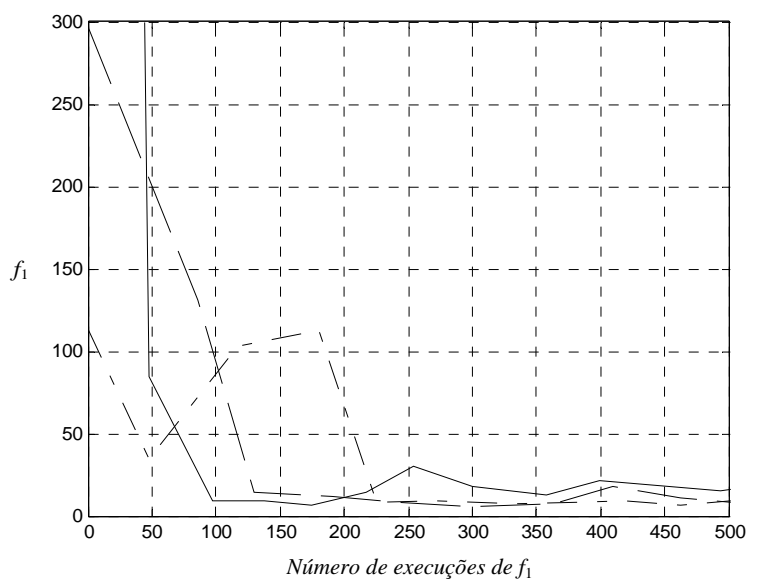

Figura 6 - Evolução da função de desempenho pelo número de execuções de $f_{1}$ (método de Rosenbrock: linha tracejada; método de Rosenbrock com recozimento simulado: linha com traços diferentes; método de Powell: linha sólida).

Valores iniciais dos parâmetros: $K=10, z=-10$ [rad/s], $p=$ $-500[\mathrm{rad} / \mathrm{s}]$.

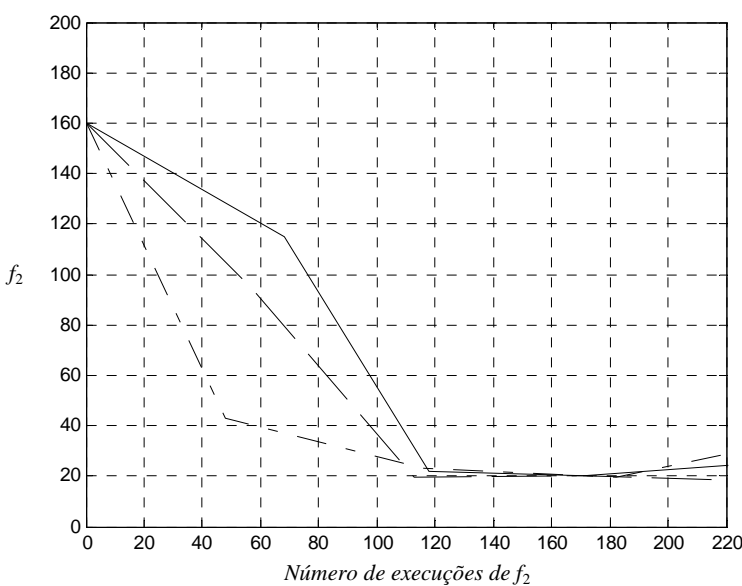

Figura 7 - Evolução da função de desempenho pelo número de execuções de $f_{2}$ (método de Rosenbrock: linha tracejada; método de Rosenbrock com recozimento simulado: linha com traços diferentes; método de Powell: linha sólida).

Valores iniciais dos parâmetros: $K=10, z=-10, p=-500$.

Qualitativamente, os três métodos (Rosenbrock, Rosenbrock com recozimento simulado e Powell) se assemelharam bastante, isto é, decréscimo rápido da função nas primeiras iterações e valor mínimo da função custo semelhante. Não se verificou, dessa forma, ganho no uso do recozimento simulado. A análise quantitativa não leva a resultados conclusivos devido à mudança dos minimizadores com o tempo.

Os critérios de parada utilizados não foram rígidos: optouse muitas vezes por interromper a busca quando o mínimo parecia ter sido atingido e o valor da função desempenho começava a oscilar.

Os valores das funções $f_{1}$ e $f_{2}$ tiveram uma evolução semelhante durante o processo de otimização. A função $f_{3}$ teve seu mínimo atingido mais rapidamente como ilustra o gráfico da figura 8. Não há como comparar os valores numéricos obtidos para cada função por seus significados diferentes.

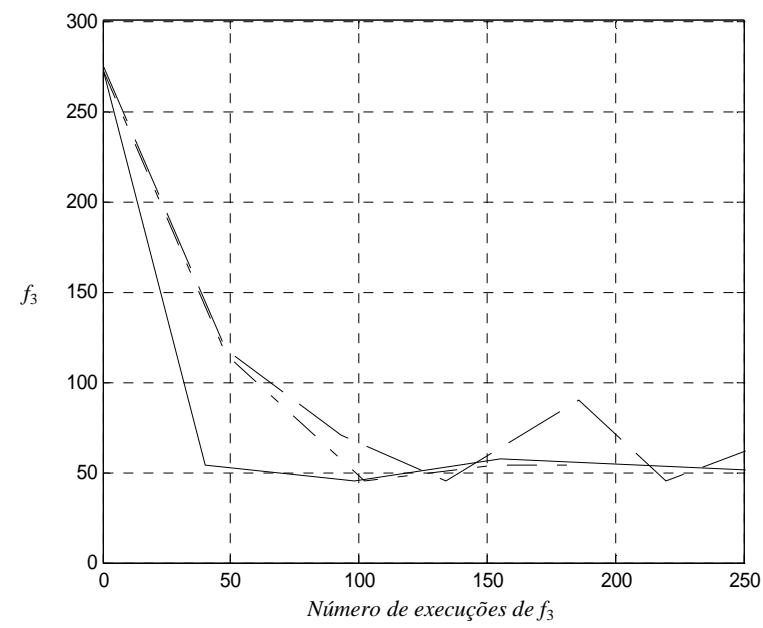

Figura 8 - Evolução da função de desempenho pelo número de execuções de $f_{2}$ (método de Rosenbrock: linha tracejada; método de Rosenbrock com recozimento simulado: linha com traços diferentes; método de Powell: linha sólida). Valores iniciais dos parâmetros: $K=10, z=-10, p=-500$.

\subsection{Métodos para Acelerar o Procedimento de Otimização}

Há duas maneiras para acelerar o procedimento de otimização: alterando o algoritmo de otimização ou a função de desempenho. A primeira opção não esteve no foco deste trabalho. Investigou-se como acelerar a otimização através da diminuição do tempo de cálculo da função de desempenho.

Um modo de fazê-lo seria utilizar um critério de acurácia que decidisse pelo cálculo ou pela interpolação da função de desempenho com base em seus valores já calculados em outros pontos. Mas tal procedimento não se aplicaria para um sistema variante no tempo.

Outra opção seria diminuir o período da onda quadrada usada para perturbar o sistema. Mas, diminuíndo esse 
período, o sistema não teria tempo para estabilização. Portanto, essa opção funcionaria apenas para sistemas físicos mais rápidos. Para esses casos, acredita-se que surgirá outra limitação, a qual residirá no tempo de transmissão das amostras do escravo para o mestre, a exemplo do que ocorreu neste mesmo trabalho nos primeiros testes do procedimento de otimização: o tempo de execução da função de desempenho era de 20 [s], o dobro daquele conseguido posteriormente, o que se devia ao fato de que a transmissão de dados do escravo para o mestre durava quase 10 [s]. O problema foi resolvido diminuindo-se o número de amostras. Acredita-se que para sistemas mais rápidos a diminuição do número de amostras poderá prejudicar a exatidão da função de desempenho. Dessa forma, uma solução possível é migrar da comunicação via interface serial para a comunicação via interface de rede, o que diminuiria o tempo de transmissão.

\section{COMENTÁRIOS E CONCLUSÃO}

Neste trabalho, o uso de técnicas de otimização de controladores com hardware na configuração mestreescravo levou ao desenvolvimento de uma estratégia de sintonia fina de controladores que, objetivando o desempenho ótimo, pode ajustar um controlador simples às condições específicas (magnetização) de um sistema à medida que essas mudam com o tempo. Esse tratamento é também especialmente interessante para a redução da amplitude de ciclos-limites tão comuns em sistemas como controladores de atitude (Oliveira e Kienitz, 2000). Naquele exemplo a sintonia fina é importante na fase pós-projeto durante uma simulação HIL ("hardware-in-the-loop").

Foram ainda propostas e desenvolvidas algumas técnicas que permitem a aceleração do processo de otimização. Essas técnicas teriam maior impacto para sistemas mais simples que o testado (sistemas invariantes no tempo e bem modelados). Características importantes dessa configuração são o seu baixo custo e a simplicidade de sua adaptação a outros sistemas físicos.

A principal dificuldade foi a ocorrência de ocasionais quedas da esfera ou sua colisão com o eletroimã durante o processo de otimização. Por um lado foi constatada a impossibilidade de obter-se modelos úteis para prever de forma confiável essas ocorrências. Por outro lado verificouse que a sintonia fina do controlador a partir de um controlador inicial razoável (possivelmente oriundo de algum projeto) é um procedimento com resultados práticos relevantes. Portanto tolerou-se tal problema e alguns algoritmos de otimização mostraram-se bem menos sensíveis à dificuldade do que outros.

Como prosseguimento a este trabalho é interessante a aplicação da metodologia aqui apresentada a outros sistemas físicos no escopo de uma ferramenta de sintonia de controladores, por exemplo aquela apresentada por Kienitz (1997).

\section{REFERÊNCIAS BIBLIOGRÁFICAS}

Feedback Instruments Ltd. (1997). Magnetic Levitation System.

Galperin, E.A. (1993). "The alpha algorithm and the application of the cubic algorithm in case of unknown Lipschitz constant," Computers Math. Applic., v. 25, no 10/11, pp 71-78.

Galvão, R.K.H.; Yoneyama, T.; de Araújo, F.M.U.; Machado, R.G. (2003) "A simple technique for identifying a linearized model for a didactic magnetic levitation system," IEEE Transactions on Education, pp. 22-25, v. 46, no 1 , 2003.

Gill, P.; Murray, W.; Wright, M.H. (1981) Practical optimization, Academic Press, London.

Grimm, C. (2002). Um Controlador Digitalmente Assistido para um Sistema de Levitação Magnética, tese de mestrado, Instituto Tecnológico de Aeronáutica, S. José dos Campos.

Himmelblau, D.M. (1972). Applied nonlinear programming, McGraw Hill, New York.

Kienitz, K.H. (1997) "A toolbox for requirements engineering and optimization-based dynamic system design from linguistic specifications", Proc. IDEA '97 (Intelligent Design in Engineering Applications), pp. 77-82, ELITE Foundation, Aachen, Alemanha.

Mateus, G.R. \& Luna, H.P.L. (1986) Programação não linear, UFMG, Belo Horizonte.

Nascimento J.R. \& T. Yoneyama (2000). "Métodos de otimização numérica," Inteligência Artificial em Controle e Automação , capítulo 9, pp. 92-106, Edgard Blücher, São Paulo.

Oliveira, N.M.F. \& Kienitz, K.H. (2000) "Attitude controller design for a system using actuators with switching-time restrictions and delays", Proceedings AIAA Guidance, Navigation, and Control Conference, Artigo AIAA-2000-3967, Denver, CO.

Powell, M.J.D. (1964) "An efficient method for finding the minimum of a function of several variables without calculating derivatives," Computer Journal, v. 7, pp. 155-162.

Rosenbrock, H.H. (1960) "An automatic method for finding the greatest or least value of a function," Computer Journal, v. 3, 175-184. 
Spendley, W.; Hext, G.R.; Himsworth, F.R. (1962) "Sequential application of simplex designs in optimization and evolutionary design," Technometrics, v. 4, pp. 441-461.

Swann, W.H. (1964) Report on the development of a new direct search method of optimization, ICI Ltd Central Instrumentation Laboratory Research Note 64/3. 